\title{
Overview of the DLR RailwayDynamics Library
}

\author{
Andreas Heckmann $^{1} \quad$ Marc Ehret $^{1} \quad$ Gustav Grether $^{1} \quad$ Alexander Keck $^{1} \quad$ Daniel Lüdicke $^{1}$ \\ Christoph Schwarz ${ }^{1}$ \\ ${ }^{1}$ Institute of System Dynamics and Control, German Aerospace Center (DLR), Germany, \\ andreas.heckmann@dlr.de
}

\begin{abstract}
The newly released commercial DLR RailwayDynamics Library is intended to support the design, optimization and control development as well as hardware- and software-inthe-loop testing of railway vehicles mainly on the system level. To this aim, it provides the capability to consider vehicle dynamics issues such as traction, comfort and safety in multi-domain engineering tasks by preparation of vehicle, track, wheel-rail contact models and roller rig scenarios on different levels of detail.

Exploiting several precursor papers on specific railway modeling topics, their models have been collected and reorganized in order to propose a sound modeling framework dedicated to railway dynamics.

The paper gives an overview on particular concepts and ideas of the library, presents several application examples and discusses two approaches to organize multi-domain modeling.

Keywords: railway vehicle dynamics, wheel-rail contact, multi-domain vehicle modeling
\end{abstract}

\section{Introduction}

\subsection{Background}

A high level of safety and comfort as well as sustainability and protection of natural resources are high-level objectives of the DLR-internal, long-term research project Next Generation Train (NGT) and compiled to vehicle dynamics, suspension design, running gear development and active guidance control as associated tasks of the DLR Institute of System Dynamics and Control (SR).

Despite the significance of hardware testing, modeling, simulation and optimization remain the dominating tools in research efforts on safety enhancements, function upgrades, comfort improvement and reduction of wear, energy consumption and life-cycle costs. These tools offer the opportunity to examine and evolve new technical concepts in early design phases without implementation risks and by comparable low costs. Moreover, the use of Modelica is in particular attractive since it provides the capability to cover multi-domain engineering tasks in one consistent simulation environment, cf. (Carrarini et al., 2010).

Hence, it is not surprising that there already exists a slew of Modelica publications that report on NGT and cooperation project results in railway engineering such as on energy flows in electric railway networks (Heckmann and Streit, 2012), on wheel-rail contact (Heckmann et al., 2014a), running gear (Schwarz et al., 2015) and pneumatic brake system modeling (Ehret, 2018) and on crosswind stability assessment (Heckmann and Grether, 2017).

\subsection{Objectives}

The idea of the present paper is to gather those models and experiences from the work quoted above, organize and propose a modeling framework dedicated to railway dynamics and running gear design. This includes the consideration of the nonlinear wheel-rail contact in normal and in tangential direction in view of the wheel and rail profile geometry.

Besides analyzing classical vehicle dynamics topics such as traction, comfort and safety, the capability to work on multi-domain engineering tasks is a specific focus of the RailwayDynamics Library. In fact, railway vehicles also employ multiphysical subsystems such as pneumatic friction brakes and air suspensions, electrical engines to provide propulsion and to regenerate energy, Diesel-electric or Diesel-hydraulic drive trains and so on.

With this background, the commercial DLR RailwayDynamics Library is supposed to support holistic system design, optimization and hardware or software in-the-loop testing by provision of vehicle dynamics models that may be scaled and adapted with respect to the required modeling level.

An overview on the library structure is given in the following section. Some particularities of railway modeling are presented in Section 3, while Section 4 contains elaborate example applications. Section 5 is a discussion on different approaches for multi-domain modeling. Section 6 concludes the paper and gives an outlook.

\section{Overview}

\subsection{Library Structure}

Figure 1 presents the main subpackages of the library. Each major subpackage and its models are additionally marked by using different fundamental icon colors, namely light grey, light green, light red or light blue.

The General subpackage contains multi-purpose models for all kind of analysis in the context of railway dynamics and also covers the operation of test rigs. It includes lateral, longitudinal, vertical, roll, pitch and yaw dynam- 


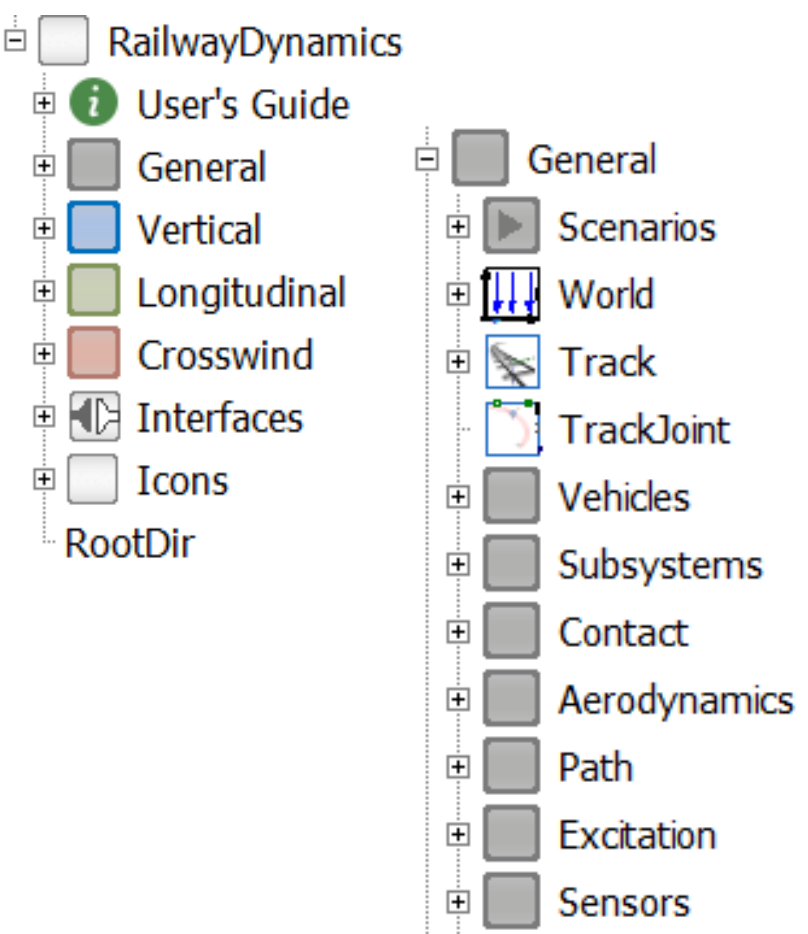

Figure 1. Structure of the RailwayDynamics Library

ics which may be investigated for comfort, traction, safety purposes among others.

The Vertical subpackage gathers specific models with vertical degrees of freedom, only, which could be used for preliminary surveys on vibration comfort and the associated lay-out of suspensions.

The Longitudinal subpackage is intended to be used to study traction and braking maneuvers of trains, which explicitly requires to consider the longitudinal motion of railway vehicles and the associated rotational motion of wheels or wheelsets, respectively.

The Crosswind subpackage is tailored for quasistatic crosswind stability analysis according to Sec. 5.4.3 in (EN 14067-6: 2010).

Generally speaking, models from the Vertical, Longitudinal or Crosswind subpackage are specializations and are supposed to replace models from the General subpackage in order to focus on more specific analysis goals and balance the computational resources according to the needs on hand.

\subsection{Vehicle Substructuring}

The example vehicle model dedicated to one single car in Figure 2 gathers submodels to represent

- the railroad base as an aggregation of track joints and track panels to be further explained in Section 3.2 and Section 3.3,

- two running gears, which include wheelsets, bogie frames, primary and secondary suspensions,

- and the carbody.

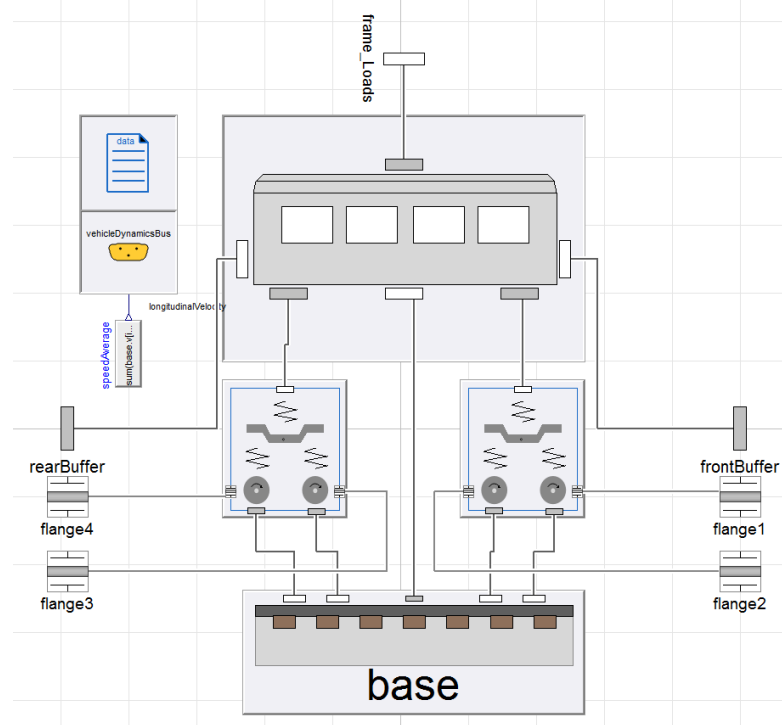

Figure 2. Structure of vehicle model

The vehicle model composition in Figure 2 serves as a template and is employed throughout the complete library. Each submodel may be replaced by another submodel that stems from the same partial base class.

Four flange with bearing connectors from the Modelica Standard Library (MSL) and its Multibody subpackage, respectively, allow for the application of traction torques from the outside to be transferred to the wheelsets and, as an option supported at the bogie frame.

Two multibody frame connectors called rear and front buffer enable the connection to leading of traveling cars.

The vehicle dynamics bus here transmits one signal which is the longitudinal velocity of the car.

Data on masses, fundamental geometry, primary and secondary suspensions are collected by one data record, to be further explained in the following section.

\subsection{Data Concept}

The collection of fundamental vehicle data in one record and their propagation to submodels is useful to retain control and a clear view on the parametrization of the model. However, almost each vehicle requires different data and needs a particular record structure since e.g. the options to design railway suspensions are numerous.

Therefore, it appears useful to declare the Data record locally as a specifically tailored encapsulated record as follows:

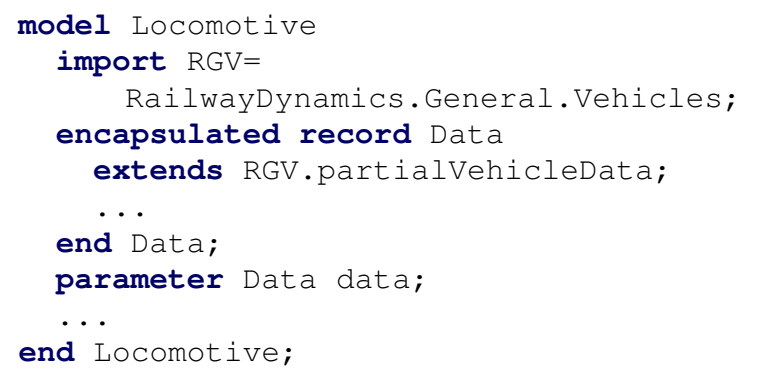


The submodels of the vehicle here called Locomotive are supposed to refer to the above declared record in the following way:

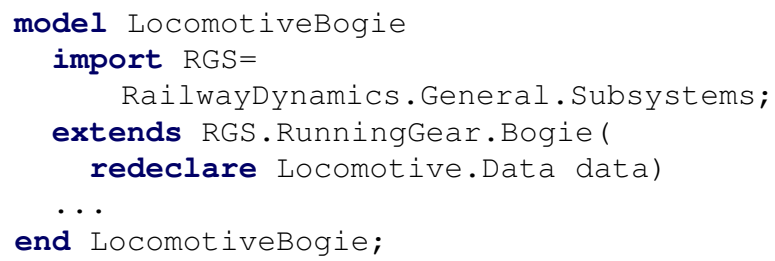

\section{Railway Modeling Particularities}

In order to introduce the particularites of the RailwayDynamics Library, Figure 3 shows a simple scenario, namely a single wheelset running along curved track.

\subsection{Track}

The track instance is mandatory for every model which uses the RailwayDynamics Library except the later on presented roller rig environment. It contains information on some global parameters using the Modelica inner/outer mechanism and defines

- the path as a function of the path length parameter $s$, i.e. the 3D curve $\boldsymbol{r}=\boldsymbol{r}(s)$, the vehicle is intended to move along, and the collateral frame, whose unit basis vectors are $\boldsymbol{t}=\boldsymbol{t}(s), \boldsymbol{n}=\boldsymbol{n}(s)$ and $\boldsymbol{b}=\boldsymbol{b}(s)$,

- the rails, which are symmetrically aligned along the path and

- the irregulartities that specify local deviations or disturbances of the idealized path and rail definition.

The 3D curve of the path is described by supporting points, which are interpolated by B-Splines in a sufficiently smooth manner. Together with the superelevation or roll angle $\phi(s)$, the supporting points are read from a

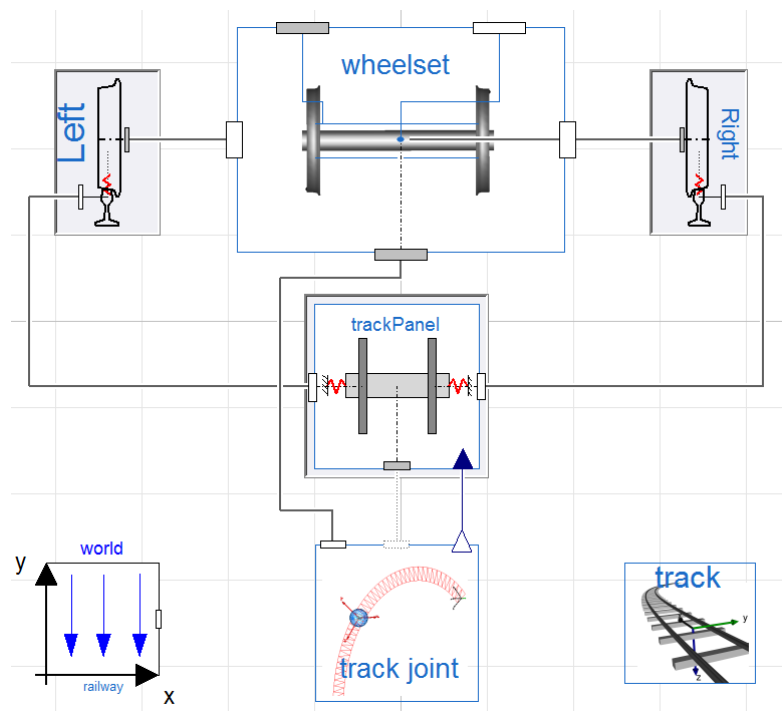

Figure 3. Diagram layer of a wheelset on a curved track model file, to which a string parameter of the track component refers. The orientation of the collateral frame then results from the following definitions:

$$
\begin{aligned}
& \boldsymbol{t}(s)=\frac{\boldsymbol{r}_{, s}}{\left|\boldsymbol{r}_{, s}\right|} \quad \text { with } \quad()_{, s}:=\frac{\partial()}{\partial s}, \\
& \boldsymbol{n}(s)=\left(\begin{array}{ccc}
1 & 0 & 0 \\
0 & \cos \phi(s) & \sin \phi(s) \\
0 & -\sin \phi(s) & \cos \phi(s)
\end{array}\right)(\boldsymbol{a} \times \boldsymbol{t}), \\
& \boldsymbol{b}(s)=\boldsymbol{t} \times \boldsymbol{n},
\end{aligned}
$$

where the auxiliary unit vector $\boldsymbol{a}$ is a user defined parameter. Its introduction is needed to overcome the shortcomings of the Frenet frame definition, which refers to $\boldsymbol{t}_{, s}$ in order to specify $\boldsymbol{n}$ and results in a zero vector for straight line paths, cf. (Weber, 1990).

In the animation in Figure 4 the path progress is delineated as a transparent red band. The red frame presents the local triad at the instantaneous position. Whenever the terms longitudinal, lateral and vertical are used in the context of this library, they refer to this local coordinate system that moves along the predefined track path. The longitudinal direction hereby is specified by $\boldsymbol{t}$, i.e. tangential to the instantaneous track position, and the vertical axis referring to $\boldsymbol{b}$ is pointing downwards as usual in railway dynamics.

The position and orientation of the two rail reference frames are given relative to the path by three parameters: gauge, gaugeOffset and inclination, for which usual values are $1 / 20$ or $1 / 40$.

The irregularities implemented so far are random realizations of disturbances with specified spectral properties. The user may select four different geometrical types of irregularities (vertical, lateral, crosslevel and gauge) from a number of predefined spectra that are taken from railway vehicle textbooks or papers, e.g. (Frederich, 1984) or (Haigermoser et al., 2015).

\subsection{Track Joint}

Recall the prismatic joint from the MSL-Multibody subpackage that specifies one mechanical degree of freedom

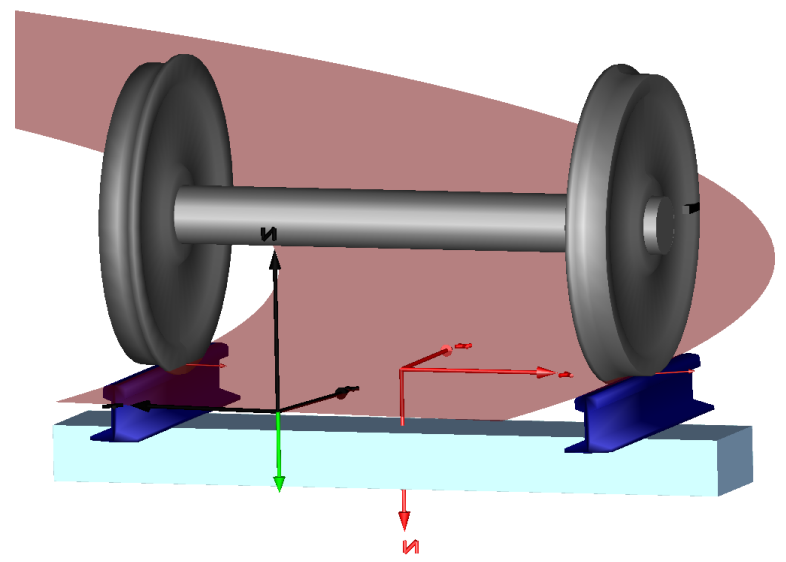

Figure 4. Animation of a wheelset on a curved track model 


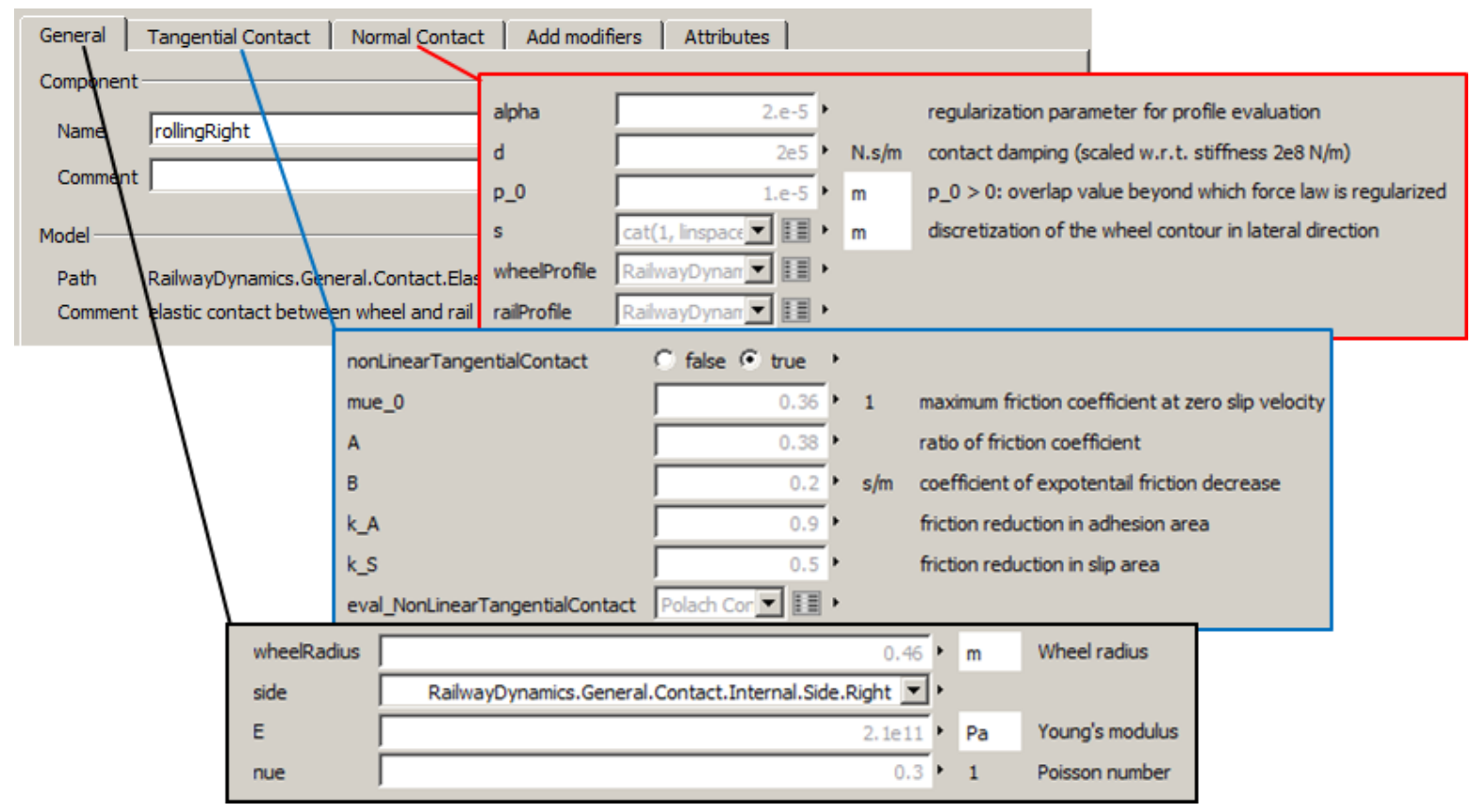

Figure 5. Excerpts from the menu to specify parameters of the elastic Contact model.

or two states, which represent the capability to move along a straight line. In the same manner, the track Joint defines one mechanical degree of freedom, but now refers to the track instance and presents the capability to move along the 3D path $\boldsymbol{r}$. The two associated states are $s_{j}=s_{j}(t)$ and $v_{j}=v_{j}(t)$, i.e. the instantaneous position along the path and its time derivative.

The local frame specified by $\boldsymbol{t}, \boldsymbol{n}$ and $\boldsymbol{b}$ uniquely assigns an orientation to each instantaneous position on the 3D track path and a given translational track speed $v_{j}=v_{j}(t)$ corresponds to a specific angular velocity $\boldsymbol{\omega}_{j}=$ $\boldsymbol{\omega}_{j}\left(v_{j}(t), \boldsymbol{r}\left(s_{j}\right)\right)$.

The railroad base in Figure 2 contains five track Joint components to represent the longitudinal degrees of freedom of the four wheelsets and the carbody.

\subsection{Track Panel}

As visualized in the animation in Figure 4, the track panel presents two rail stubs and one sleeper that are assumed to move in longitudinal direction associated to the wheelset. This is a quite common model simplification in railway dynamics in order to avoid the representation of the rail and the subgrade structure as a distributed system with many degrees of freedom and high computational demands. The flexibility of the rail road may then be introduced by modeling the track panel as a discrete springdamper-mass system, which is parametrized on the basis of shaker measurements (Chaar and Berg, 2006). Thus, the mutual influence of neighbouring track panels through the rails and the subgrade is neglected.

Since the vehicle model considers four wheelsets, the railroad base in Figure 2 contains four track panel components, which are connected using vectors of MSL Multibody frames.

\subsection{Wheelset}

The wheelset model reproduces interia properties and contains two prismatic joints to enable lateral and vertical motion and three revolute joints to allow for roll, yaw and revolute motion. These five degrees of freedom complement the longitudinal motion already covered by the attached track Joint.

\subsection{Wheel-Rail Contact}

The wheel-rail contact component, which here is elastic Contact, is to be connected to the wheelset multibody frame that is located at the axle bearing position and the rail profile reference frame of the track panel. Figure 5 shows the General dialog menu tab, where wheel radius, Young's modulus, Poisson number and a side flag have to be specified.

The Normal Contact tab in Figure 5 specifies

- the smoothing parameter $\alpha$, which is associated to a proposal of Arnold et al. to even profile curvature jumps, see (Arnold and Netter, 1998) or (Heckmann et al., 2014a),

- a contact damping parameter $d$,

- a parameter $p_{0}$ that helps to regularize the Hertzian contact algorithm, see (Heckmann and Grether, 2017),

- a vector $\boldsymbol{s}$ of lateral positions that samples the wheel contour in a number of discrete points,

- a reference to the wheel and rail profile geometry in the following manner:

import RailwayDynamics.General. Contact; 


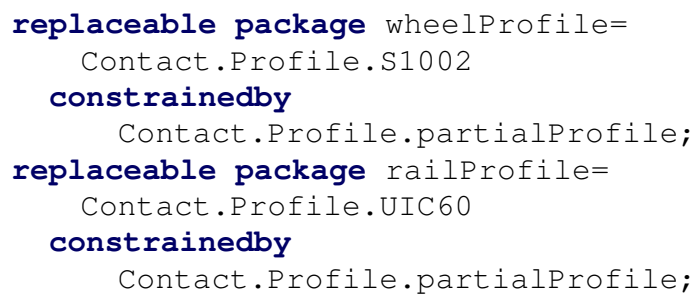

These two profile packages may be replaced by the user, so that other standard or even measured profiles may be introduced, as long as the following base class is inherited:

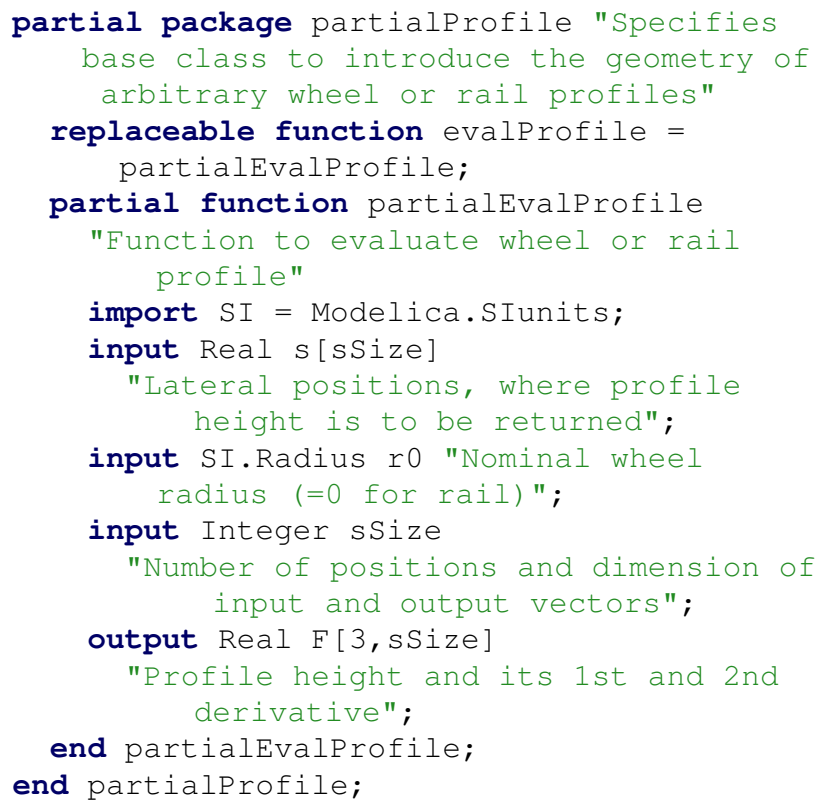

The Tangential Contact tab allows for

- switching between linear and nonlinear tangential contact evaluation,

- refering to a replaceable function to evaluate the nonlinear contact and may be user-defined as well,

- specifying parameters associated to the predefined nonlinear contact formulation according to Polach, see (Polach, 2005) or (Heckmann et al., 2014a).

In order to replace the ElasticContact in Figure 3, a Constraint contact model as described in (Heckmann et al., 2014a) and a Simplified contact component, which considers the wheel to present a conical profile that runs along a sharp edge rail are available in the library. The Normal Contact tab for this alternative contact models slightly differs to what is described above.

In addition, contact model classes to represent the rolling contact of a wheel to a roller as given in roller rigs are available in die subpackage RailwayDynamics.General.Contact. All contact models implemented so far consider the wheel and rail profile to touch each other at just one single point and exploit the usual assumptions in multibody vehicle modeling collected in Table 1 of (Heckmann et al., 2014a).

\section{Example Applications}

\subsection{Traction}

The goal of traction analysis is to calculate the acceleration and resulting in-train forces as well as longitudinal oscillations of coupled vehicles during traction and braking maneuvers. These are important investigations regarding safety, longitudinal comfort, fatigue of components, train control, vehicle stability and energy considerations, cf. (Spiryagin et al., 2014).

In Figure 6 an industrial scaled train model consisting of a locomotive and 4 cars is pictured. The vehicles are connected by coupling elements. The model is used to simulate the acceleration and deceleration phase of the train driven by the locomotive and to estimate the resulting in-train forces. A simple control unit sets the torques of the wheelsets of the locomotive in order to reach the target velocity.

In order to minimize the computational effort for this kind of simulations the Longitudinal subpackage offers models with a reduced number of lateral and vertical degrees of freedom. The train model in Figure 6 consists of models from the General subpackage (car1, grey) and from the Longitudinal subpackage to be distinguished by their light green icon fill color (locomotive and car2,3,4). In the longitudinal vehicle models the relative motions between carbody and bogie as well as between bogie and wheelset, except for the rotation of the wheelset, are neglected and therefore no suspension elements between these bodies are applied. Furthermore, the excitation caused by track irregularities is ignored. However, the vehicle model moves along the 3-D path defined by the track model and is therefore affected by downhill-slope forces and resistance forces caused by curvature of the track. The calculated velocity of the locomotive and the resulting intrain forces acting in couplerl are shown in Figure 7 (assembly1). One can observe peaks of the coupler forces caused by the impact of the cars on each other at the beginning of the acceleration and brake phases.

In order to compare the simulation results and the computational effort using models from the different subpack-

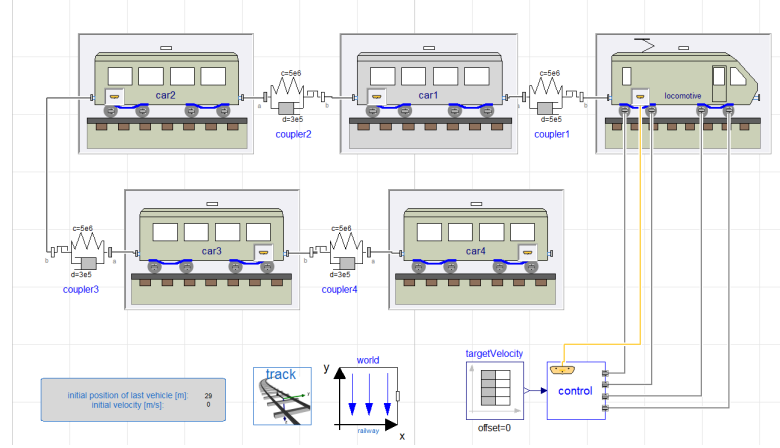

Figure 6. Mixed model built up of locomotive and cars from the General and the Longitudinal subpackage to simulate traction and braking maneuver (assemblyl) 


\begin{tabular}{|c|c|c|}
\hline assembly & CPU-second / second & number of states \\
\hline assembly1 & 6.65 & 157 \\
\hline assembly2 & 0.08 & 51 \\
\hline assembly3 & 69.50 & 605 \\
\hline
\end{tabular}

Table 1. Table comparing computational effort and time states of three different model assemblies

ages, two more assemblies of the introduced train are built up and simulated. In assembly2 all vehicle models of the train are from the Longitudinal subpackage and in assembly3 all vehicle models are from the General subpackage. Figure 7 compares the simulation results of the three different model assemblies. It illustrates that the simulated vehicle speed and the peaks of the simulated coupler forces of all three model assemblies coincide.

A comparison of the computation time per simulated second and the number of continuous time states is shown in Table 1. The train model built up from the Longitudinal subpackage only (assembly2) leads to a simulation model with 51 states that computes the results within $8 \mathrm{~s}$. The replacement of car1 by a model from the General subpackage, as shown in Figure 6 (assembly 1), leads to 157 states and a computation time of $665 \mathrm{~s}$. The computation of assembly 2 using models from the General subpackage only takes $6950 \mathrm{~s}$ and uses 605 states.

This example demonstrates that the Longitudinal subpackage provides suitable models which are capable to analyze the longitudinal dynamics of trains by requiring only a fraction of the computational effort compared to a simulation of the train considering the entire vehicle dynamics. Furthermore, the models of this subpackage allow the analysis of very long trains, such as freight trains with up to 200 cars, with reasonable computational effort.

However, it is important to be aware of the limited scope of simulations using models of the Longitudinal subpackage. Due to the reduced number of degrees of

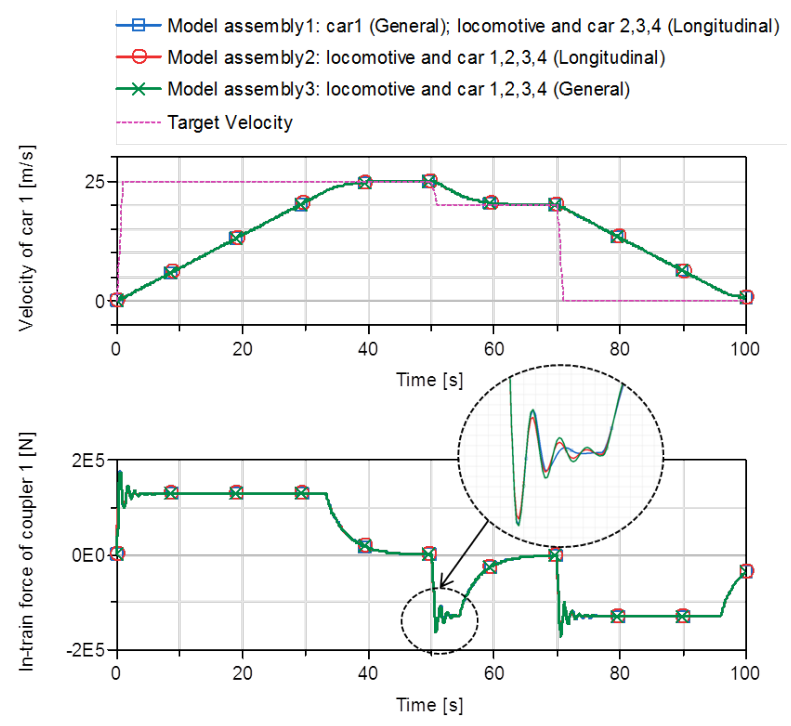

Figure 7. Comparison of simulation results of models from the Longitudinal subpackage and the General subpackage freedom certain dynamics, such as pitch, yaw and roll of the bogie and carbody cannot be simulated. Thus, the simulation of scenarios in which this behavior might influence traction or braking of vehicles need to be carried out by using models that take these dynamics into account. A potential scenario is the reduction of traction or brake forces induced by pitching of the bogie which in turn decreases the normal contact forces between wheel and rail and in consequence the maximum transferable traction force.

The replacement strategy in Figure 6 also allows for mixed scenarios e.g. with one vehicle model from the General subpackage surrounded by several ones from the Longitudinal subpackage. The General model then provides a detailed insight, while the Longitudinal models are mainly intended to introduce the interaction with neighboring cars.

\subsection{Comfort}

As all technical components rails are non-ideal systems and exhibit irregularities, which induce vehicle vibrations. These track excitations are characterized by distance frequency components in power density spectra (PSD). In the RailwayDynamics Library the usual PSDs of ERRI and Frederich as well as own PSDs can be defined. Usual passenger trains therefore use two-level suspensions to reduce vibrations of the carbody to meet ride comfort targets. The lay-out of these suspensions is a significant engineering task, which can be done by simulation using full vehicle models from the General subpackage.

However in early design phases, it is a common assumption that the ride comfort is dominated only by vertical vibrations. This premise opens the opportunity to simplify vehicle models and reduce their computational needs in order to facilitate optimization studies. To this aim, the Vertical subpackage of the RailwayDynamics Library provides quarter and half vehicle models.

The example model of a quarter vehicle in Figure 8 consists of a track panel, a bogie and a car body model. The

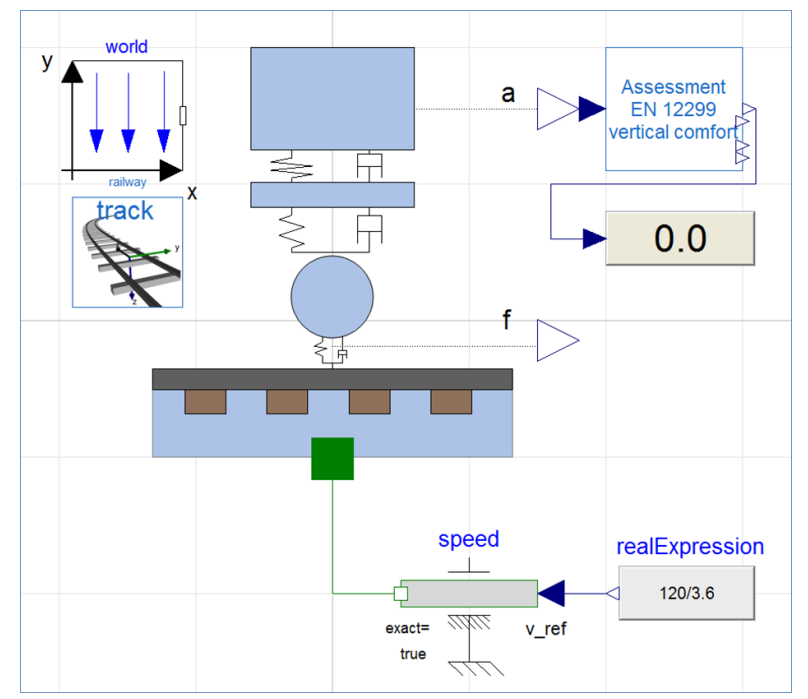

Figure 8. Vertical quarter vehicle model 
position of the vehicle on the track is determined by an external input to the translational flange connector, since the longitudinal dynamics is neglected.

As mentionend in Section 3.3 the track panel is presented as a discrete sleeper mass, supported by a springdamper system. The position of the rails follows the motion of the track panel, to which predefined irregularities are superimposed, cf. Section 3.1. The wheel-rail contact model here is simplified to a spring-damper system. The primary suspension that connects wheel and bogie mass and the secondary suspension between bogie and carbody mass are also spring-damper models with one vertical degree of freedom, each.

Several calculation methods can be used to express the human comfort perception of a rail vehicle in a performance index. The evaluation of the vibration comfort of a rail vehicle is defined in the (EN 12299: 2009) standard. The average comfort is expressed with the $N_{M V}$ value, where the acceleration measurement is reshaped by various frequency filters, so that the $N_{M V}$ number quantitatively expresses the human sensation of vibration comfort. To be more specific, values $N_{M V}<1.5$ are characterized as very comfortable while values $N_{M V} \geq 4.5$ are assessed to be very uncomfortable.

In a first application example, an AVMZ-wagon, see (Iwnicki, 1998) runs at $120 \mathrm{~km} / \mathrm{h}$ on a track with excitation. The vertical vibration comfort $N_{M V z}$ is determined on the basis of a full-vehicle model from General subpackage and a quarter vehicle model shown in Figure 8.

The track irregularities are characterized by a customized polynomial with input parameters $a_{i}$ and $b_{i}$ :

$$
S(\Omega)=\frac{b_{0}+b_{2} \Omega^{2}}{a_{0}+a_{2} \Omega^{2}+a_{4} \Omega^{4}+a_{6} \Omega^{6}}, f=\frac{\Omega}{2 \pi}\left[\frac{1}{m}\right] .
$$

Figure 9 shows the PSD $S(f)$ of the customized excitation as polynomial (blue) that is compared to two PSDs from literature called ERRI high and ERRI low (Bergander and Kunnes, 1993). For verification purposes, the customized irregularities were additionally measured from the simulation results and their PSD was reconstituted in cyan in Figure 9. As indicated by the signal drop at the

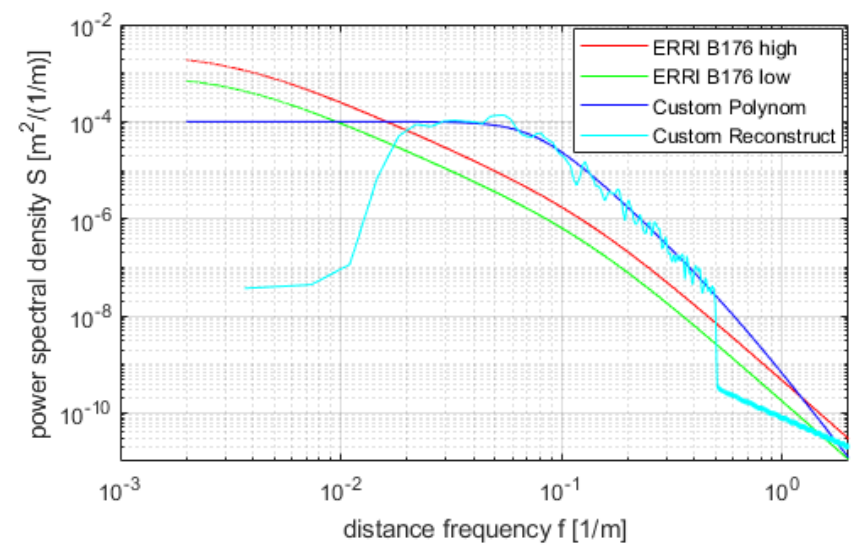

Figure 9. Comparison of track irregularities defined by power spectral densities (PSD) edges of the reconstituted PSD, the customized excitation has been limited to $0.02 \leq f \leq 0.5[1 / \mathrm{m}]$ by user input.

The vibration comfort of the full vehicle model is different along the carbody due to the contribution of its pitch motion to the local accelerations. While it is best in the middle, it is worst at the vehicle ends. The simulated vibration comfort of the quarter vehicle model shows an average comfort of the full-vehicle model. Reviewing the number of states in Table 2, it has to be taken into account that the various frequency filters according to (EN 12299: 2009) introduce 31 states into both model assemblies. The following table compares the simulation results of the two vehicle models:

\begin{tabular}{|c|c|c|c|}
\hline Vehicle Model & states & CPU-s/s & $N_{M V z}$ \\
\hline full vehicle & 171 & 9.08 & $0.43 \ldots 0.83$ \\
\hline quarter vehicle & 40 & 1.58 & 0.63 \\
\hline
\end{tabular}

Table 2. Table comparing computational effort and time states of two different model assemblies

\subsection{Roller Rig}

The use of test rigs for railway research and development is widely spread in industry as well as at research institutes, see e.g. (Jaschinski et al., 1999). Even if test rigs cannot entirely replace track tests, in early design phases a test rig provides essential benefits, like cost effectiveness, repeatable testing conditions and an extended set of measurement equipment. To support this testing process the General subpackage contains all necessary components including specific contact models for the wheel-roller contact to build up a suitable simulation environment.

An animation of a model of a single wheelset on a roller rig is shown in Figure 11, to which Figure 10 presents the diagram layer. The so-called uFrame in green in Figure 11

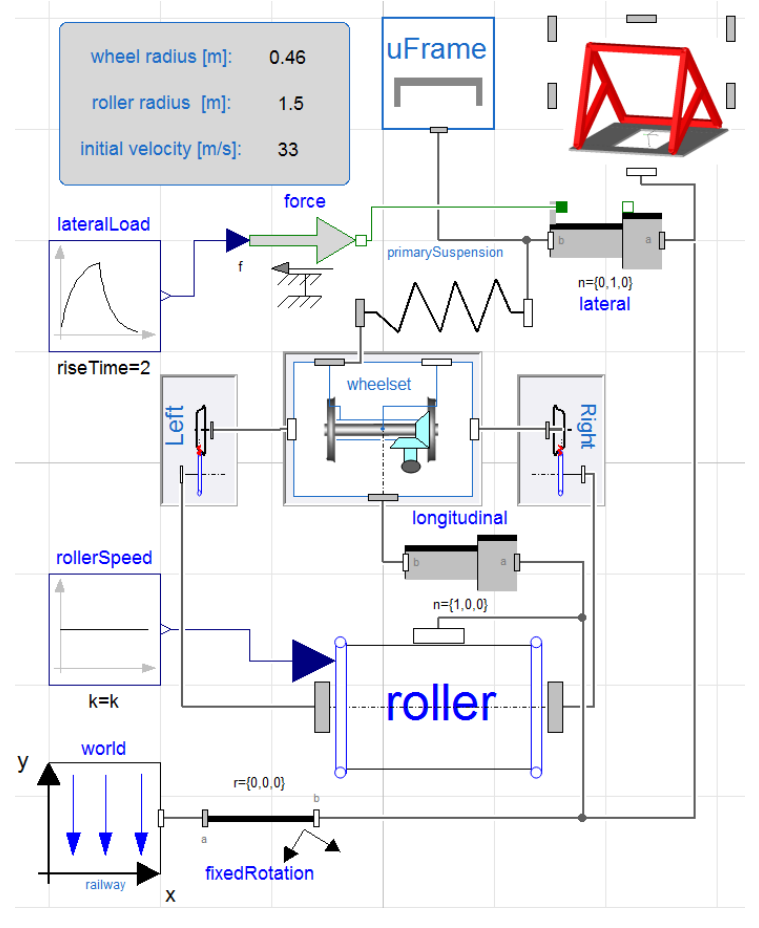

Figure 10. Diagram layer of a roller rig with one wheelset 


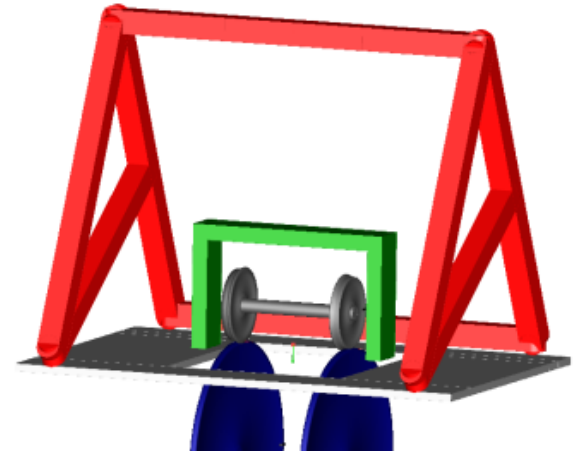

Figure 11. Animation of a roller rig with one wheelset

imitates the bogie while the aFrame in red represents the carbody in a simplified way.

If longitudinal investigations are of interest, the test rig can be extended by brake units and traction motors, respectively, to validate for example new wheel slide protection and anti-skid algorithms.

Besides the longitudinal analysis the roller rig environment can be used to evaluate the lateral dynamics, what is exemplarily presented in Figure 12. The two pictures result from a test rig simulation with a lateral force excitation (blue line in the upper plot) on the uFrame. The red line denotes the resulting force in the primary spring, which clearly shows the interdependence of the higher frequency wheel-roller contact forces.

In the lower plot the lateral displacements of the wheelset (green) and the uFrame (black) are illustrated and the typical hunting motion can be recognized especially by the wheelset behavior. The difference between these two signals is the relative deviation of the primary spring. In the end, this scenario allows to verify or even optimize the dynamic stability of the wheelset e.g. under a crosswind disturbance or an other lateral influence.
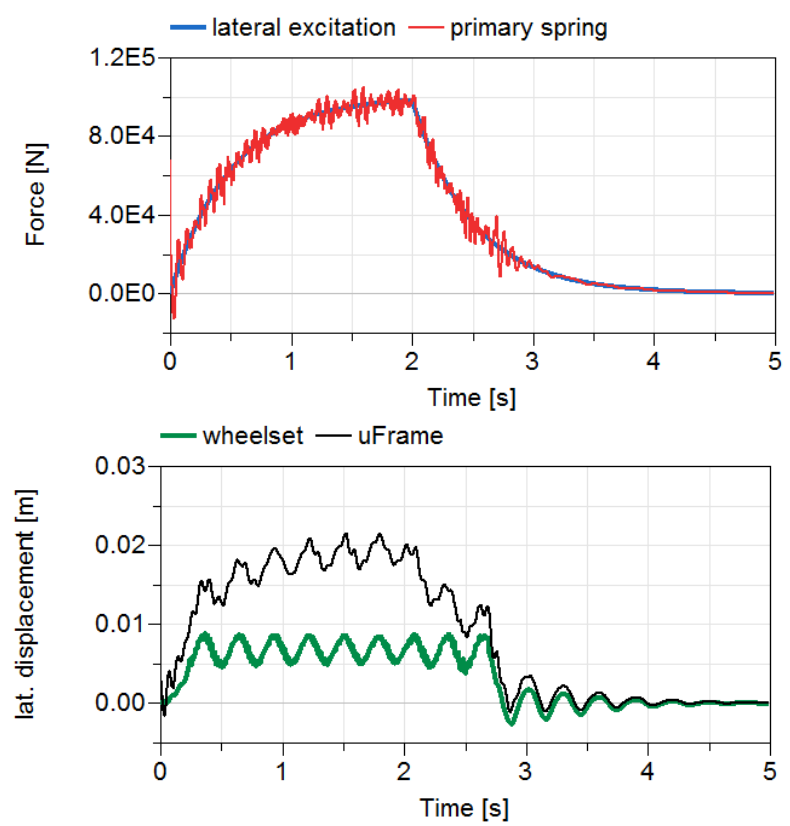

Figure 12. Simulation results of a laterally excited wheelset on the roller rig

\subsection{Crosswind Stability}

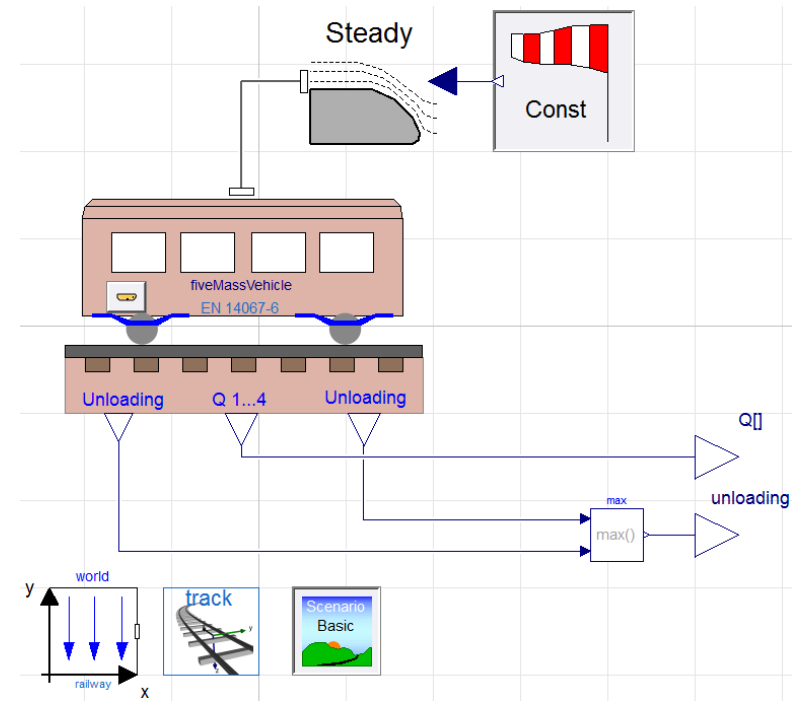

Figure 13. Diagram layer of the quasistatic crosswind model according to EN 14067-6

Crosswind stability addresses the risk that vehicles running on high speed are prone to overturning, if high crosswinds occur. The assessment of this risk is part of the vehicle acceptance procedure, regulated by (TSI HS RST 2008) and (EN 14067-6: 2010). One of several assessment scenarios defined in these references refers to a simplified five-mass model, which is therefore predefined in the RailwayDynamics Library. Figure 13 shows the diagram layer of this quasistatic model. The reader is referred to (Heckmann and Grether, 2017), where a detailed discussion on the crosswind stability issue and further modeling approaches are given.

\section{Multi-domain Modeling}

\subsection{The VehicleInterfaces Library reloaded}

As already mentioned in Section 1.2, the RailwayDynamics Library is intended to facilitate the multi-domain modeling of railway vehicles. Therefore, the present paper makes the attempt to initiate a discussion on the appropriate organization of multi-domain railway vehicle models. The multi-domain railway model in Figure 14 is inspired

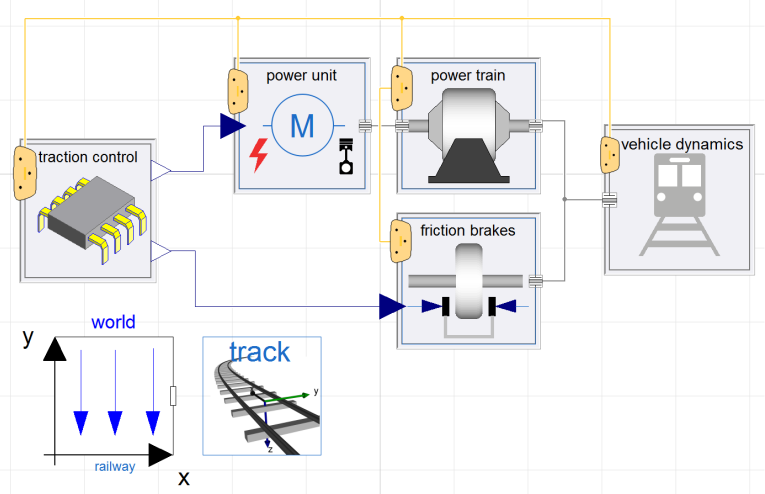

Figure 14. First option to organize multi-domain modeling 


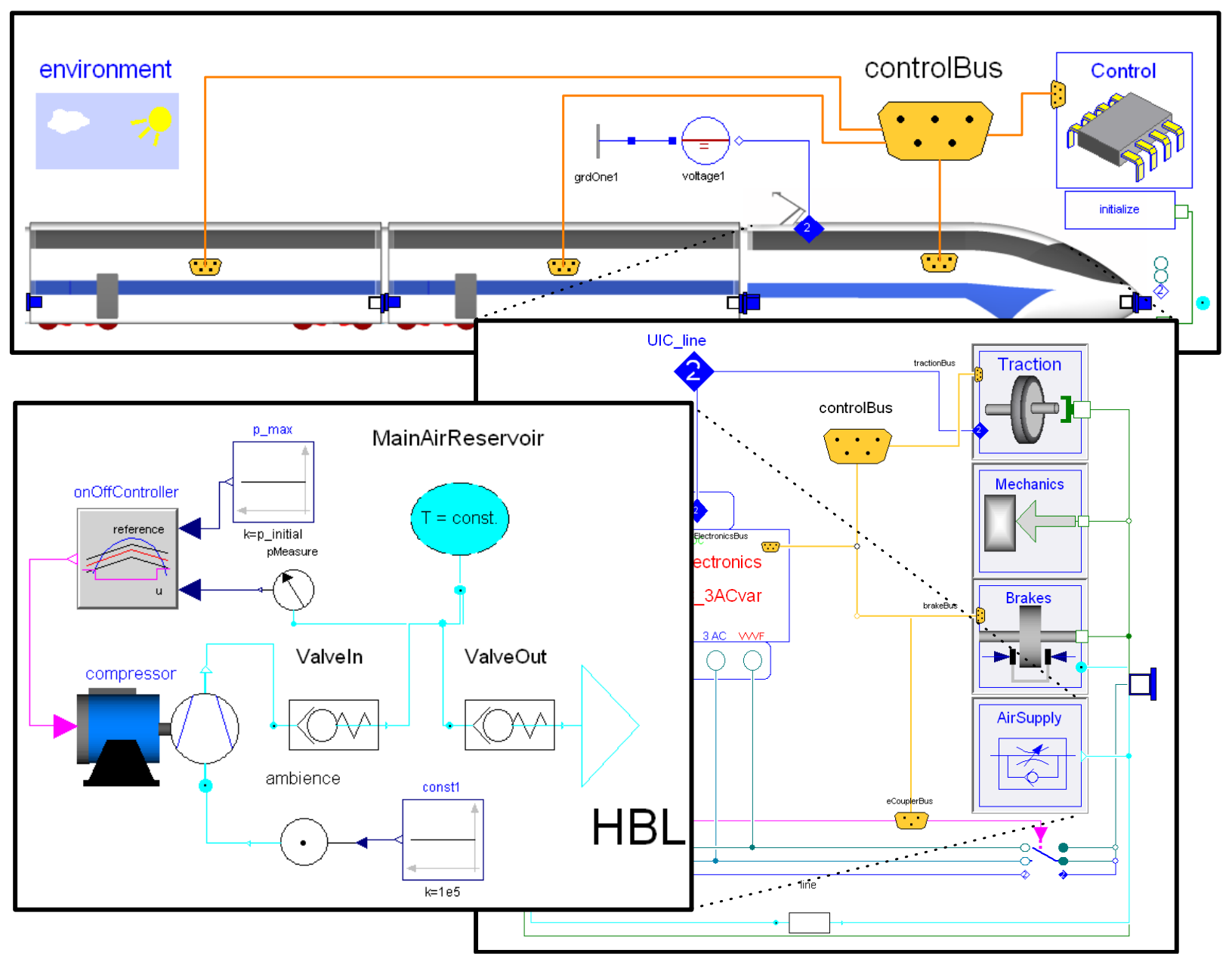

Figure 15. Alternative option to organize multi-domain modeling from (Carrarini et al., 2010)

by a corresponding activity in the automotive field that led to the definition of the VehicleInterfaces library (Dempsey et al., 2006).

There, the issues on railway vehicle dynamics are presented by one submodel depicted by one icon. Additional submodels cover propulsion systems, power train and brake modeling and introduce control algorithms. Following this scheme, it is easy to replace submodels e.g. in order to adapt their detail level. External supplier companies may provide submodels of their domain and the interconnection of the submodels may be organized on the model top level.

However, railway vehicles actually are train sets, where several cars are connected at buffers. Each single car is a multiphysical system on its own. Therefore, the scheme in Figure 14 actually presents an aggregation of singledomain train sets, one vehicle dynamics train set, one train set of propulsion systems, one for brakes, etc. A vector of flange with bearing connectors propagates traction or braking torques to the wheelsets, cf. Figure 2.

\subsection{Alternative Approach}

Figure 15 originates from a former project proposal for auxiliary systems in trains. There, each car is a multi- domain model with (electric) traction, air supply, mechanical and brake subsystem. The component view of the air supply subsystem is shown in Figure 15 as well. The list of optional subsystems may be further extended, e.g. to consider the energy supply of air conditioning subsystems or the control of door systems.

A newly specified multi-domain buffer connector was defined that connects single cars, e.g. by connecting the pneumatic line of the leading car with the pneumatic line of the trailing one and so on.

This approach is assumed to rely on a more elaborate specification of all stakeholders such as OEMs and suppliers on interfacing and multilevel modeling organization compared to the proposal in Section 5.1. The multidomain overhead an engineer has to keep in view while working on his or her single-domain task might be larger. The authors of the present paper are curious where a discussion on this issue may lead to.

\section{Summary and Outlook}

This paper presents the newly released DLR RailwayDynamics Library, which is intended to provide a sound modeling framework dedicated to vehicle dynamics and running gear design. The consideration of vehicle dynamics 
issues in multi-domain engineering tasks is a specific focus of the RailwayDynamics Library.

Already initiated and future applications of the library concern the synthesis of advanced observer and control lay-outs, (Schwarz et al., 2018), (Heckmann et al., 2016), and multidisciplinary simulation tasks such as the interaction of running dynamics and drive train, the systems engineering of pneumatic brake systems (Ehret, 2018) and research on advanced system design and assessment scenarios in order to ensure the crosswind stability of railway vehicles, cf. (Heckmann et al., 2014b).

\section{Acknowledgment}

A very early version of the subpackage of the RailwayDynamics Library that is specialized to crosswind stability was implemented by Dr. Antonio Carrarini during his period of employment at DLR.

\section{References}

M. Arnold and H. Netter. Approximation of contact geometry in the dynamical simulation of wheel-rail. Mathematical and Computer Modelling of Dynamical Systems, 4(2):162-184, 1998.

B. Bergander and W. Kunnes. ERRI B176/DT 290: B176/3 Benchmark Problem, Results and Assessment. Technical report, European Rail Research Institute, 1993.

A. Carrarini, A. Heckmann, I. Kaiser, B. Kurzeck, J.L.R̃eyes Pérez, and L. Valente. Multidisciplinary applications of multibody simulation to railway vehicle engineering. In IMSD 2010, 2010. URL https://elib.dlr.de/ $64436 /$.

N. Chaar and M. Berg. Simulation of vehicle-track interaction with flexible wheelsets, moving track models and field tests. Vehicle System Dynamics, 44(sup1):921-931, 2006. doi:10.1080/00423110600907667.

M. Dempsey, M. Gäfvert, P. Harman, C. Kral, M. Otter, and P. Treffinger. Coordinated automotive libraries for vehicle system modelling. In Proceedings of the 5th International Modelica Conference, Vienna, pages 33-41, 2006.

M. Ehret. Modelca library for the systems engineering of railway brakes. In Proceedings of the American Modelica Conference 2018, 2018.

EN 12299: 2009. Railway Applications -Ride comfort for passengers - measurement and evaluation, 2009.

EN 14067-6: 2010. Railway Applications -Aerodynamics- Requirements and test procedures for crosswind assessment., 2010 .

F. Frederich. Die Gleislage aus fahrzeugtechnischer Sicht. ZEVGlasers Annalen, pages 108-1984, 1984.

A. Haigermoser, B. Luber, J. Rauh, and G. Gräfe. Road and track irregularities: measurement, assessment and simulation. Vehicle System Dynamics, 53(7):878-957, 2015. doi:10.1080/00423114.2015.1037312.
A. Heckmann and G. Grether. The DLR RailwayDynamics Library: the Crosswind Stability Problem. In Proceedings of the 12th International Modelica Conference, pages 623-631, 2017. doi:10.3384/ecp17132623.

A. Heckmann and S. Streit. The modeling of energy flows in railway networks using xml-infrastructure data. In Proceedings of the 9th International Modelica Conference, pages 125-132, 2012. doi:10.3384/ecp12076125.

A. Heckmann, A. Keck, I. Kaiser, and B. Kurzeck. The Foundation of the DLR RailwayDynamics Library: the Wheel-Rail-Contact. In Proceedings of the 10th International Modelica Conference, pages 465-475, 2014a. doi:10.3384/ECP14096465.

A. Heckmann, B. Kurzeck, T. Bünte, and S. Loose. Considerations on active control of crosswind stability of railway vehicles. Vehicle System Dynamics, 52(6):759-775, 2014b. doi:10.1080/00423114.2014.901539.

A. Heckmann, C. Schwarz, T. Bünte, A. Keck, and J. Brembeck. Control development for the scaled experimental railway running gear of DLR. In 24th Symposium of the International Association for Vehicle System Dynamics (IAVSD 2015). CRC Press, 2016.

S. Iwnicki. The Manchester Benchmarks for rail simulators - an introduction. Vehicle System Dynamics, 29(sup1):717-722, 1998. doi:10.1080/00423119808969598.

A. Jaschinski, H. Chollet, S. Iwnicki, A. Wickens, and J. Würzen. The application of roller rigs to railway vehicle dynamics. Vehicle System Dynamics, 31(5-6):345-392, 1999.

O. Polach. Creep forces in simulations of traction vehicles running on adhesion limit. Wear, 258(7):992-1000, 2005. doi:10.1016/j.wear.2004.03.046.

C. Schwarz, A. Heckmann, and A. Keck. Different models of a scaled experimental running gear for the DLR RailwayDynamics Library. In Proceedings of the 11th International Modelica Conference, pages 441-447, 2015. doi:10.3384/ecp15118441.

C. Schwarz, J. Brembeck, and B. Heckmann. Dynamics observer for the longitudinal behavior of a wheelset on a roller rig. Proceedings of the Institution of Mechanical Engineers, Part F: Journal of Rail and Rapid Transit, 2018. submitted for publication.

M. Spiryagin, C. Cole, Y.Q. Sun, M. McClanachan, V. Spiryagin, and T. McSweeney. Design and simulation of rail vehicles. CRC Press, 2014. doi:10.1201/b17029.

TSI HS RST 2008. 2008/232/EC: Commission Decision of 21 February 2008 concerning a technical specification for interoperability relating to the rolling stock sub-system of the trans-european high-speed rail system, 2008.

W. Weber. Die Gleisbogenachse als räumliches Kurvenstück. ETR, 39(1/2):79 - 81, 1990. 